\title{
Compositional Ordering and Polar Nano-Regions: Physical Effects of Sn Alloying in $\mathrm{SrTiO}_{3}$ Thin Films
}

\author{
Everett D. Grimley ${ }^{1}$, Tianqi Wang ${ }^{2}$, Bharat Jalan ${ }^{2}$, and James M. LeBeau ${ }^{1}$
}

1. Department of Materials Science and Engineering, North Carolina State University, Raleigh, USA.

2. Department of Chemical Engineering and Materials Science, University of Minnesota, Minneapolis, USA.

The properties accessible to perovskite oxides can be substantially modified through solid-solution alloying, which can even produce altogether new functionality. Recent theoretical studies, for example, have suggested that the excellent ferroelectric properties in $\mathrm{Pb}^{2+}$ lead-based ferroelectrics might be alternatively obtained by stabilizing $\mathrm{Sn}$ as $\mathrm{Sn}^{2+}$ in a perovskite [1]. Towards this end, heavily alloyed $\mathrm{Sn}: \mathrm{SrTiO}_{3}$ thin films were recently synthesized, and exhibited signatures of relaxor ferroelectricity in second harmonic generation experiments [2]. Relaxor-like ferroelectricity is usually thought to result from the formation of the polar nano-regions coupled to other changes in the crystal lattice, which are readily measureable with high-angle annular dark-field scanning transmission electron microscopy (HAADF STEM) [3].

In this talk, we explore the local changes in composition, strain, and non-centrosymmetric displacements in $\mathrm{Sn}$ alloyed $\mathrm{SrTiO}_{3}$ thin films with accurate and precise STEM imaging. We will demonstrate inhomogeneity in the films consistent with compositional ordering and formation of polar nano-regions. Revolving STEM imaging [4] (see Figure 1a) directly reveals that Sn-rich and Sn-poor Ti-sites tend to cluster in patches in the $\mathrm{Sn}: \mathrm{SrTiO}_{3}$ on the (001) Ti growth planes. Furthermore, this clustering is readily identified in maps of the column intensities (see Figure 1b) and shows good correlation to local tetragonality of the sample (see Figure 1c), where the color corresponds to the out-of-plane/in-plane lattice parameter (c/a) ratio. Furthermore, displacements of the Ti-sites from the cell centers are typically larger in the $\mathrm{Sn}: \mathrm{SrTiO}_{3}$ than in the $\mathrm{SrTiO}_{3}$ substrates (see Figure 1d), indicating likely breaking of inversion symmetry as needed for the observed relaxor properties.

Since $\mathrm{Sn}$ is expected to occupy the perovskite lattice as $\mathrm{Sn}^{2+}$ or $\mathrm{Sn}^{4+}$ on the $\mathrm{Sr}$ - or Ti-sites respectively, we will discuss results from STEM energy-dispersive X-Ray spectroscopy (EDS) and quantitative analysis of HAADF STEM atom column intensities. EDS reveals that $\mathrm{Sn}$ primarily incorporates on the Ti-site (see Figure 2a), though the poor Sn signal makes it challenging to directly assess Sr-site occupancy. Histograms of experimental HAADF atom column intensity also supports primarily Ti-site incorporation. Sr- and Ti-site intensities from pure and $\mathrm{Sn}$-alloyed $\mathrm{SrTiO}_{3}$ show marked change for Tisites with little-to-no change for the Sr-sites (see Figure 2b). To understand the role of Sn on image intensities, we will discuss results from multislice image simulations conducted using $\mathrm{SrTiO}_{3}$ supercells with different $\mathrm{Sn}$ at $\%$ on the Sr-sites and Ti-sites. These simulations indicate that Sn incorporated on Srand Ti-sites introduces distinguishable changes to the intensity distributions (see Figure 2c). Finally, the correlation between atomic structure, chemistry, and polarization will be discussed [5]. 
References:

[1] SF Matar, I Baraille and MA Subramanian, Chemical Physics 355 (2009) p. 43.

[2] T Wang et al, APL Materials 4 (2016) p. 126111.

[3] X Sang et al, Applied Physics Letters 106 (2015) p. 061913.

[4] X Sang and JM LeBeau, Ultramicroscopy 138 (2014) p. 28.

[5] NC State Univ. (NCSU) and Univ. of Minnesota authors acknowledge support from the U.S.

National Science Foundation (NSF) through Grants DMR-1350273 and DMR-1607318, respectively. EDG acknowledges support from the NSF Graduate Research Fellowship Program (DGE-1252376).

Work was performed in part at the Analytical Instrumentation Facility (a member of RTNN, a site of NNCI) at NCSU, which is supported by the State of NC and the NSF (award number ECCS-1542015).

\section{a) HAADF-STEM}

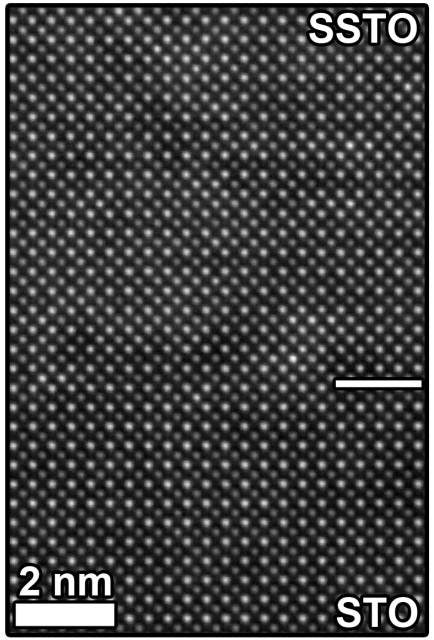

b) Column Intensity

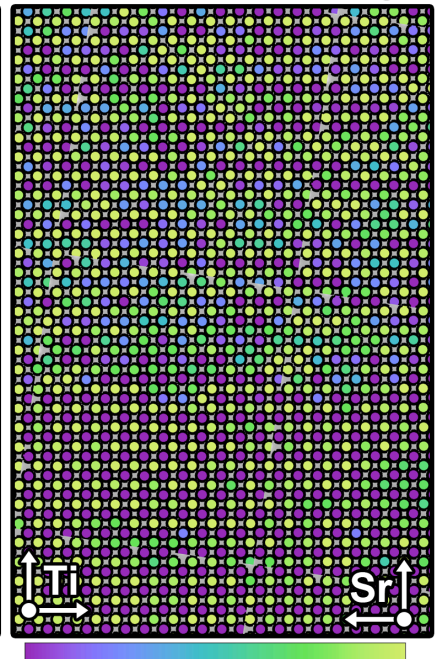

Intensity (arb.) c) c/a Ratio

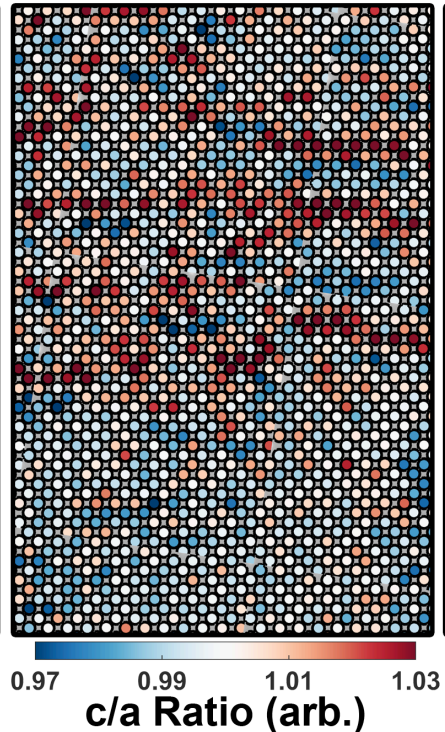

d) Ti-site Displ.

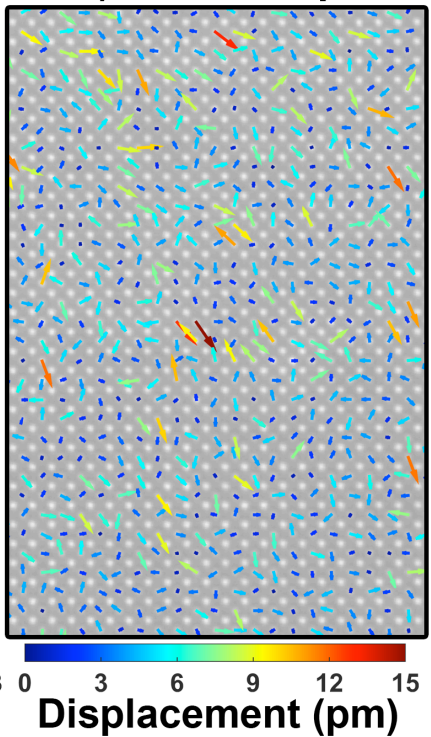

Figure 1. a) $\mathrm{Sn}: \mathrm{SrTiO}_{3} / \mathrm{SrTiO}_{3} \mathrm{HAADF}$ RevSTEM ([100] zone axis) with maps of b) intensity, c) c/a ratio, and d) Ti-site displacement. Color scales are set to enhance details over the appropriate ranges.

a) EDS Mapping

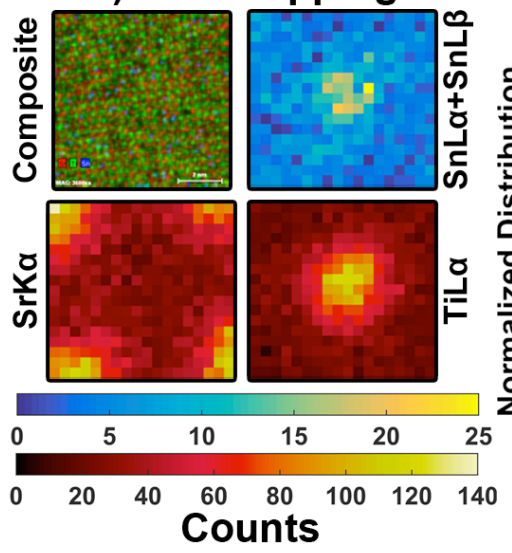

b) Experimental Column Int.

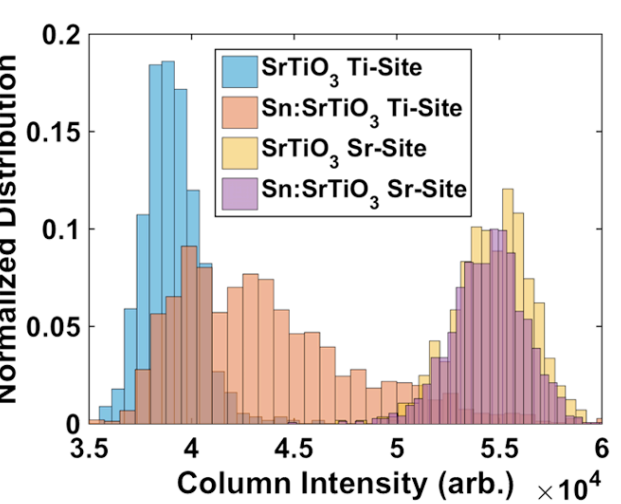

c) Simulated Column Int.

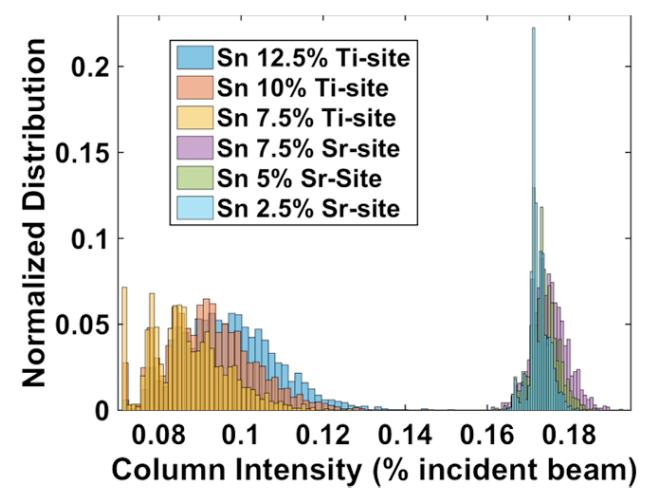

Figure 2. a) Atomic resolution STEM-EDS of $\mathrm{Sn}: \mathrm{SrTiO}_{3}$ with template averaged signals (note separate $\mathrm{Sn}$ and $\mathrm{Sr} / \mathrm{Ti}$ color scales). b) Histogram of experimental Sr- and Ti-site atom column intensities from $\mathrm{Sn}: \mathrm{SrTiO}_{3}$ compared to $\mathrm{SrTiO}_{3}$ substrate. c) Histogram of column intensities taken from simulations of randomly alloyed supercells where the quantity of Sr- and Ti-site atoms replaced with Sn are given. 